Pacific Journal of Mathematics

DUALITY AND ASYMPTOTIC SPECTRAL DECOMPOSITIONS 


\title{
DUALITY AND ASYMPTOTIC SPECTRAL DECOMPOSITIONS
}

\author{
RIDGLEY LANGE
}

\begin{abstract}
Asymptotic spectral decomposition for an operator on a Banach space is studied in light of the well-known theory of decomposable operators of Foias type. It is proved that adjoints of strongly quasidecomposable operators have the single-valued extension property. Duality theorems for strongly decomposable operators are given, for example, an operator has strongly decomposable adjoint iff it has a rich supply of strongly analytic subspaces. For reflexive spaces sharper results are obtained. Decomposable operators are characterized as those quasi-decomposable operators satisfying an additional duality property. Also an asymptotic spectral decomposition with strongly analytic subspaces implies decomposability. Strongly bi-decomposable operators are also studied.
\end{abstract}

1. Introduction. The theory of decomposable operators, initiated by Foias [11], and given substantial development by him and others in the period 1963-1975, can now be said to have reached a satisfactory maturity. It is known, in particular, that decomposable operators enjoy a "completely symmetric duality theory," i.e., an operator is decomposable exactly when its adjoint is. It has also been proven by the author [20] and independently by E. Albrecht [3] and B. Nagy [23] that decomposable operators form the widest class of operators having "linear spanning" spectral decompositions (see below). (For other special properties of decomposable operators, see [5].)

There has also been parallel development of spectral theory along the separate but related path of "asymptotic decompositions." To be precise, let $T$ be a bounded linear operator on the Banach space $X$. We say that $T$ has an asymptotic spectral decomposition if, for each finite open cover $\left\{G_{i}\right.$ : $1 \leq i \leq n\}$ of the complex plane, there is a system of $T$-invariant subspaces $\left\{M_{1}, M_{2}, \ldots, M_{n}\right\}$ such that

(ASD) $\quad X=\bigvee_{i=1}^{n} M_{i}$ and $\sigma\left(T \mid M_{i}\right) \subset G_{i}$ for $i=1, \ldots, n$.

Our notation is standard: $X$ is the closed span of $\left\{M_{l}\right\}$. 
By imposing further restrictions on the class of invariant subspaces that can be used in the (ASD), we may achieve different classes of operators. We survey briefly those classes that have been studied extensively. An operator $T$ is decomposable (D) if each $M_{\imath}$ in (ASD) is spectral maximal and the $M_{i}$ span $X$ linearly:

$$
X=M_{1}+M_{2}+\cdots+M_{n} .
$$

(A $T$-invariant subspace $M$ is spectral maximal if for any $T$-invariant subspace $N$ the inclusion $\sigma(T \mid N) \subset \sigma(T \mid M)$ implies $N \subset M$.) In $\S 2$ we give the most important facts of this theory. If for each spectral maximal space $M$ it happens that the restriction $T \mid M$ is also decomposable, we say $T$ is strongly decomposable (SD) [4]. Obviously (SD) $\Rightarrow(\mathrm{D})$.

Colojoară and Foias remark in the appendix of their monograph on decomposable operators [7] that a weakening of condition (1) to (ASD) (this is the historical order) might lead to successful generalizations. In his 1974 thesis, A. Jafarian [15] undertook this study of so-called weakly decomposable operators and did indeed prove many analogs of the earlier theory. But since at that time many important questions on decomposable operators were still open, there has been little progress in the (ASD) theory. By 1981 these questions had largely been answered; in particular, Frunză [13] and Liu and Wang [22] proved the necessity and sufficiency, resp., of the theorem: $T$ is decomposable iff its adjoint is (see Corollary 1). One aim of the present paper is to take up again the question of duality for the (ASD). We shall see that in general the problem is complicated even in the case of reflexive spaces.

Let us recall that $T$ is weakly decomposable (WD) if the subspaces in (ASD) are spectral maximal. The author [17] studied still another class called analytically decomposable operators (AD). An operator $T$ is in class (AD) if the invariant subspaces in the (ASD) are taken to be analytically invariant [14] (see §3 for a definition). Frunză proved [14] that every spectral maximal space is analytically invariant, hence (WD) $\Rightarrow(A D)$. Combining all of the implications above, we obtain the chain (SD) $\Rightarrow$ (D) $\Rightarrow(\mathrm{WD}) \Rightarrow(\mathrm{AD})$. The converses of the first two of these implications are false. Albrecht gave counterexamples to the first and second of these in [2] and [1], resp. Whether $(\mathrm{AD}) \Rightarrow(\mathrm{WD})$ is still an open question. In the following section, we give the chief facts on decomposable operators, both for reference in later sections and as an illustration of how well-behaved decomposable operators are. In what follows, $T$ will always be a bounded linear operator on the complex Banach space $X$ with respective adjoints $T^{*}$ and $X^{*}$. If $M$ is a $T$-invariant subspace, we write $T \mid M$ for the 
restriction and $T^{M}$ for the operator induced by $T$ on the quotient $X / M$. For $M \subset X$, let $M^{\perp}$ be its annihilator in $X^{*}$. We use $\sigma(T)$ for spectrum of $T$ and $\rho(T)$ for its resolvent set, and we let $\mathbf{C}$ denote the (finite) complex plane. The complement of $A$ in $B$ is written $B-A$, and we put $\bar{A}$ or $A^{-}$for the closure of $A$ in an appropriate topology.

\section{Decomposable operators.}

THEOREM 1. For an operator $T$, the following are equivalent.

(i) $T$ is decomposable.

(ii) Thas an (ASD) with arbitrary subspaces; and (1) is satisfied.

(iii) For every open set $G$ in $\mathbf{C}$, there is a T-invariant subspace $M$ such that $\sigma(T \mid M) \subset \bar{G}$ and $\sigma\left(T^{M}\right) \subset \mathbf{C}-G$.

Proof. The equivalence (i) $\Leftrightarrow$ (iii) was proven in [21], p. 403, while (1) $\Leftrightarrow$ (ii) was proven in three different ways in [3], [20] and [23]. (Actually, (iii) implies that $T$ is 2-decomposable, but then it is decomposable by [24].)

Assertion (ii) is remarkable because in order to prove an operator decomposable, one can bypass the need for spectral maximal spaces. We now illustrate the utility of condition (iii). First, we note that a close examination of the proof of (iii) $\Rightarrow$ (i) in [21], p. 403, reveals that it is sufficient for decomposability to establish (iii) for open sets $G$ in $\mathbf{C}$ for which $\mathbf{C}-G=(\mathbf{C}-\bar{G})^{-}$. With this reduction, we can easily prove the following corollary.

Corollary 1. $T$ is decomposable if and only if $T^{*}$ is.

Proof. Suppose that $T$ is decomposable and $G$ is open and satisfies the restriction above. Since $T$ satisfies (iii) by Theorem 1 , let $M$ be a $T$ - invariant subspace of $X$ such that $\sigma(T \mid M) \subset \mathbf{C}-G$ and $\sigma\left(T^{M}\right) \subset \bar{G}$. Hence by the usual duality relations $\sigma\left(T^{*} \mid M^{\perp}\right) \subset \bar{G}$ and $\sigma\left(T^{* M \perp}\right) \subset \mathbf{C}$ - $G$. Again by Theorem $1, T^{*}$ is decomposable. To obtain the converse, we note that by [21, Lemma 6] for decomposable $T^{*}$ each of its spectral maximal spaces $M$ in $X^{*}$ is weak* closed. From this it follows that $M=N^{\perp}$ for some $T$-invariant subspace $N \subset X$. The rest of the proof follows as in the converse case.

In the case of strongly decomposable operators, the sufficiency of the analog of Corollary 1 may fail. Recently, S. Wang [27] has announced the existence of a strongly decomposable dual operator $T^{*}$ whose predual $T$ 
is not strongly decomposable. See also Theorem 4 below in connection with this problem. In general, the duality question for larger classes of the (ASD) is still open (see §3).

3. Asymptotic decompositions. All the operators mentioned in the Introduction have the single-valued extension property (svep). An operator $T$ has the svep if for each $X$-valued analytic function $f$ defined on $V \subset \mathbf{C}$ such that $(\lambda-T) f(\lambda)=0$ for $\lambda \in V$, we have $f \equiv 0$ on $V$. The svep allows for the definition of spectral manifold for $T$. We let $X_{T}(F)$ be the set of all $u \in X$ such that $u=(\lambda-T) f(\lambda)$ for some (necessarily unique) analytic function $f: \mathbf{C}-F \rightarrow X$. Then $X_{T}(F)$ is a $T$-invariant linear manifold in $X$ [7, p. 2]. If $T$ is decomposable and $F$ is closed in $\mathbf{C}$, then $X_{T}(F)$ is norm closed in $X$ : moreover, in this case $X_{T}(F)$ is spectral maximal such that $\sigma\left(T \mid X_{T}(F)\right) \subset F$. If $T$ is weakly decomposable (WD), then it is not known that $X_{T}(F)$ is closed even if $F$ is, but a (WD) operator $T$ for which this is the case is called quasi-decomposable (QD) [15]. In fact, Albrecht's example (WD) $\nRightarrow(D)$ [1] is in the class (QD), thus (QD) must be supplemented with extra hypotheses to achieve (D). One such case is the following.

THeOREM 2. Let $T$ be quasi-decomposable. If for each open $G \subset \mathbf{C}$ the spectral inclusion

$$
\sigma\left(T^{*} \mid X_{T}(G)^{\perp}\right) \subset \mathbf{C}-G
$$

holds, then $T$ is decomposable.

Proof. By duality relations it is easy to see that the spectrum of the operator induced by $T^{*}$ on $X^{*} / X_{T}(G)^{\perp}$ is contained in $\bar{G}$. By Theorem 1 (iii) and the remark following its proof it follows that $T^{*}$ is decomposable, hence so is $T$ by Corollary 1 .

We now see that in Albrecht's example it must happen that property (2) fails for at least one $G$. Hence the adjoint of a quasi-decomposable operator is in general not decomposable. Can anything be said in the general case? To this question we have the following answer.

Proposition 1. Let $T$ be quasi-decomposable. Let $G$ be open and put $M=X_{T}(G)^{\perp}$. Then

(i) $\sigma\left(T^{* M}\right) \subset \bar{G}$ and

(ii) for each $\lambda \in G, \lambda-T^{*}$ is injective on $M$. 
Proof. Part (i) follows from the usual duality relations and the fact that $\sigma\left(T \mid X_{T}(G)^{-}\right) \subset \bar{G}$ (since $X_{T}(\bar{G})$ is closed). Let $\lambda \in G$ and let $\bar{D} \subset G$ be a disc with center $\lambda$. Then $G$ and $H=\mathbf{C}-\bar{D}$ cover $\mathbf{C}$, hence $X=X_{T}(G)^{-} \vee X_{T}(H)^{-}$. Let $u \in M$ such that $\left(\lambda-T^{*}\right) u=0$. For $x_{2} \in$ $X_{T}(H)^{-}$, the bijectivity of $\lambda-T$ on $X_{T}(H)^{-}$implies that there is $x_{2}^{\prime} \in$ $X_{T}(H)^{-}$such that $x_{2}=(\lambda-T) x_{2}^{\prime}$. Hence

$$
\left\langle x_{2}, u\right\rangle=\left\langle(\lambda-T) x_{2}^{\prime}, u\right\rangle=\left\langle x_{2}^{\prime},\left(\lambda-T^{*}\right) u\right\rangle=0
$$

It is clear that $\left\langle x_{1}, u\right\rangle=0$ for each $x_{1} \in X_{T}(G)^{-}$, hence since all such sums $x_{1}+x_{2}$ are dense in $X$, we see $u \in X^{\perp}$, or $u=0$. This proves (ii).

To get a stronger conclusion than Proposition 1, we must strengthen slightly the hypotheses on $T$ of class (QD). We say $T$ is strongly quasi-decomposable (SQD) if each restriction to $X_{T}(F), F$ closed, is also quasi-decomposable.

For our next theorem, we also require the notion of an analytically invariant subspace (in the sense of Frunza [14]). A $T$-invariant subspace $M$ is analytically invariant if for any analytic function $f: V \rightarrow X$ such that $(\lambda-T) f(\lambda) \in M$ for all $\lambda \in V$ we have also $f(\lambda) \in M$ for $\lambda \in V$. Frunza proved that $M$ is analytically invariant iff $T^{M}$ has the svep [14, p. 1062].

TheORem 3. Let $T$ be in class (SQD). Then for each open $G$ in $\mathbf{C}$, the subspace $X_{T}(G)^{\perp}$ is analytically invariant for $T^{*}$.

Proof. Clearly $X_{T}(G)^{\perp}$ is $T^{*}$-invariant since $X_{T}(G)$ is a $T$-invariant linear manifold. Let $W=X_{T}(G)^{\perp}$ and let $M=X_{T}(G)^{-}$, the norm closure in $X$. Suppose $f: V \rightarrow X$ is analytic on $V$ such that $\left(\lambda-T^{*}\right) f(\lambda) \in W$ for all $\lambda \in V$, and we may further suppose that $V$ is connected. Since the hypothesis guarantees that $X_{T}(\bar{G})$ is closed, it follows that $\sigma(T \mid M) \subset \bar{G}$. Now first assume that $V \cap(\mathbf{C}-\bar{G}) \neq \varnothing$, and let $D$ be an open disc in this intersection. For fixed $\lambda \in D$ and for all $u \in M$, we have the duality relation

$$
\langle(\lambda-T) u, f(\lambda)\rangle=\left\langle u,\left(\lambda-T^{*}\right) f(\lambda)\right\rangle=0 .
$$

Since $\lambda-T$ is bijective on $M$, we have $f(\lambda) \in M^{\perp}=W$ for $\lambda \in D$, hence $f(\lambda) \in W$ for all $\lambda \in V$ by analytic continuation. This proves that $W$ is analytically invariant in this case. Next, suppose that $V \subset \bar{G}$, hence $V \subset G$ since $V$ is open. It is clear that there are two open sets $H_{1}$ and $H_{2}$ such that $G=H_{1} \cup H_{2}$ and $V \cap\left(G-\bar{H}_{i}\right) \neq \varnothing$ for $i=1,2$. By the 
previous part of the proof, the range of $f$ lies in each $X_{T}\left(H_{i}\right)^{\perp}(i=1,2)$, hence in $X_{T}\left(H_{1}\right)^{\perp} \cap X_{T}\left(H_{2}\right)^{\perp}$. We finish the proof by showing that $W$ is precisely this intersection. Jafarian has shown that if $T$ is (SQD), it obeys the asymptotic decomposition [15, Theorem 8.3]

$$
M=X_{T}(G)^{-}=X_{T}\left(H_{1}\right)^{-} \vee X_{T}\left(H_{2}\right)^{-}
$$

for $G=H_{1} \cup H_{2}$ (all open), hence

$$
W=M^{\perp}=\left[X_{T}\left(H_{1}\right)^{-} \vee X_{T}\left(H_{2}\right)^{-}\right]^{\perp}=X_{T}\left(H_{1}\right)^{\perp} \cap X_{T}\left(H_{2}\right)^{\perp},
$$

and the proof is complete.

CoROllary 2. If $T$ is strongly quasi-decomposable, then $T^{*}$ has the suep.

Proof. It follows from Theorem 3 that if $G \supseteq \sigma(T)$, then $X_{T}(G)^{\perp}=$ $X^{\perp}=(0)$ in $X^{*}$ is analytically invariant. This is precisely the fact that $T^{*}$ has the svep.

Hence every (SQD) operator has an adjoint with the svep, so the manifolds $X_{T^{*}}^{*}(F)$ are defined, but we do not know if they are closed. In any case, since Albrecht's example of a quasi-decomposable operator which is not decomposable is actually (SQD), then its adjoint has nontrivial spectral manifolds.

We have already remarked in $\$ 2$ that the predual question for a strongly decomposable operator was answered no, i.e. $T^{*}(\mathrm{SD})$ does not imply $T(\mathrm{SD})$, but the converse question is still open. Shulberg has shown [25, Prop. 3.6, p. 151] that if $M$ is spectral maximal for the strongly decomposable $T$, then $T^{*} \mid M^{\perp}$ is decomposable. In Theorem 4 we show that there are many more decomposable restrictions of $T^{*}$.

THEOREM 4. Let $T$ be strongly decomposable. Let $G$ be an open set in $\mathbf{C}$ and let $M=X_{T}(G)^{-}$. Then $T^{*} \mid M^{\perp}$ is decomposable if $G$ and $M$ satisfy

(i) $(\sigma(T)-\bar{G})^{-}=\sigma(T)-G \neq \varnothing$, and

(ii) $\sigma(T \mid M)=\sigma(T) \cap \bar{G}$.

The proof of Theorem 4 uses the following lemma.

LEMMA 1. Let $T$ be decomposable and let $M$ and $G$ satisfy (i) above. Then

$$
\sigma\left(T^{M}\right)=[\sigma(T)-\sigma(T \mid M)]^{-}
$$


Proof. Since clearly $\sigma(T \mid M) \subset \bar{G}$, we have $\sigma(T)-\bar{G} \subset \sigma(T)-$ $\sigma(T \mid M)$. By [8, Prop. 2.2, p. 15] $\sigma(T)-\sigma(T \mid M) \subset \sigma\left(T^{M}\right)$, and by [21, Theorem 1 (6)] $\sigma\left(T^{M}\right) \subset \sigma(T) \subset \sigma(T)-G$. We thus have the chain of inclusions

$$
(\sigma(T)-\bar{G})^{-} \subset[\sigma(T)-\sigma(T \mid M)]^{-} \subset \sigma\left(T^{M}\right) \subset \sigma(T)-G,
$$

and so (3), is true if the first and last members of $(*)$ are equal. But this is the case by hypothesis (i).

REMARKs. Note that (3) fails if $\sigma(T)-\bar{G}=\varnothing$; e.g., let $T$ be multiplication by the independent variable on $C[0,1]$. Then $\sigma(T)=[0,1]$. If $G$ is the open disc with center zero and radius 1 , then $\sigma(T)=\sigma(T \mid M)=\varnothing$ and $\sigma\left(T^{M}\right)=\{1\}$. Moreover, in the statement of Theorem 3 we may assume $G \cap \sigma(T) \neq \varnothing$, for otherwise the conclusion is vacuously true.

Proof of Theorem 4. We prove that $T^{M}$ is decomposable, for then $T^{*} \mid M^{\perp}$ is decomposable by Corollary 1 . Let $H$ be an open set. We shall show that there is $N \subset X / M$ such that

$$
\begin{gathered}
\sigma\left(T^{M} \mid N\right) \subset \bar{H} \\
\sigma\left(\left(T^{M}\right)^{N}\right) \subset \mathrm{C}-H,
\end{gathered}
$$

for then Theorem 1(iii) is applicable. Without loss of generality we may suppose $H \cap \sigma\left(T^{M}\right) \neq \varnothing$, so that $\bar{H}-\bar{G} \neq \varnothing$. Let $L=X_{T}(\bar{H} \cup \bar{G})$. We claim that $N=L / M$ is the desired subspace. If we put $S=T \mid L$ then it is easy to see that $M=L_{S}(G)^{-}$( $S$ is decomposable by hypothesis). First note that $\left(T^{M}\right)^{N}=\left(T^{M}\right)^{L / M}=T^{L}$. By [21, Theorem 1(4)],

$$
\sigma\left(T^{L}\right) \subset \mathbf{C}-(H \cup G) \subset \mathbf{C}-H,
$$

so (4b) is proved. Since $T \mid L$ is decomposable, as in the proof of Lemma 1 we get

$$
\sigma(T \mid L)-\bar{G} \subset \sigma(T \mid L)-\sigma(T \mid M) \subset \sigma(T \mid L)^{M} \subset \sigma(T \mid L)-G .
$$

We claim $[\sigma(T \mid L)-\bar{G}]=\sigma(T \mid L)-G$. If the left side is properly contained in the right side, let $\lambda$ lie in $\sigma(T \mid L)-G$ but not in $[\sigma(T \mid L)-\bar{G}]^{-}$. It is then easy to see (since $\sigma(T \mid L)-\bar{G} \neq \varnothing$ ) that the distance from $\lambda$ to $\sigma(T \mid L)-G$ is positive, which contradicts the choice of $\lambda$. Hence $\sigma\left((T \mid L)^{M}\right)=[\sigma(T \mid L)-\sigma(T \mid M)]^{-}$. Now $(T \mid L)^{M}=T^{M}\left|(L / M)=T^{M}\right| N$, so

$$
\begin{aligned}
\sigma(T \mid N) & =[\sigma(T \mid L)-\sigma(T \mid M)]^{-} \subset\{(\bar{H} \cup \bar{G}) \cap \sigma(T)-\bar{G} \cap \sigma(T)\} \\
& \subset(\bar{H} \cap \sigma(T))^{-} \subset \bar{H}
\end{aligned}
$$


where we have used hypothesis (ii) and the obvious inclusion $\sigma(T \mid L) \subset$ $\sigma(T) \cap(\bar{G} \cup \bar{H})$. This proves (4a), hence the theorem follows by Theorem 1(iii).

The next theorem gives a criterion for a strongly decomposable dual operator, but first we need the notion of strongly analytic subspace [18] which is closely related to the following condition: We say $T$ has property ( $\beta$ ) $[6$, p. 394] if

for every sequence of analytic functions $f_{n}: D \rightarrow X$ such that $(\lambda-T) f_{n}(\lambda) \rightarrow 0$ uniformly (in norm) on compact sets in $D$ it follows that $f_{n} \rightarrow 0$ uniformly on compact sets in $D$.

Since J. Snader has recently proved that $M$ is strongly analytic iff $T^{M}$ has property $(\beta)$ [26], we shall say that $M$ is strongly analytic if $T^{M}$ satisfies $(\beta)$. Let us also recall that Foias proved that every decomposable operator satisfies $(\beta)$ [12, Prop. 1]. Our theorem now runs thus.

Theorem 5. The adjoint $T^{*}$ is strongly decomposable iff $T$ is decomposable and $X_{T}(G)^{-}$is strongly analytic for each open $G$.

Proof. Let $T^{*}$ be strongly decomposable. By Corollary $1 T$ is decomposable and $X_{T}(G)^{\perp}, G$ open, is a spectral maximal space of $T^{*}$ (see [13]). Put $M=X_{T}(G)^{-}$, so that $(X / M)^{*}=M^{\perp}$ (in the sense of isomorphism). But $T^{*}$ is decomposable on $M^{\perp}$ by assumption, hence $T^{M}$ is decomposable by Corollary 1 . By [12] $M$ is strongly analytic. Since $G$ was arbitrary, the necessity is proved.

Conversely, with the notation as above, we shall prove that if $M$ is strongly analytic for decomposable $T$, then $T^{*}$ is decomposable on $M^{\perp}$. It suffices by Corollary 1 to prove that $T^{M}$ is decomposable. Let $\left\{H_{1}, H_{2}\right\}$ be an open cover of $\mathbf{C}$. Then it is obvious that $X=X_{T}\left(\bar{H}_{1}\right)+X_{T}\left(\bar{H}_{2}\right)+$ $M$. Let $K_{i}=\left(X_{T}\left(\bar{H}_{i}\right)+M\right)^{-}$and $L_{i}=K_{i} / M(i=1,2)$. Since obviously $X / M=L_{1}+L_{2}$, we need only prove $\sigma\left(T^{M} \mid L_{i}\right) \subset \bar{H}_{i}(i=1,2)$ in order to apply Theorem 1(ii). Fix $i=1$ or 2 . Since $T^{M}$ has the svep, we need only prove that $\lambda-T^{M}$ is surjective on $L_{1}$ if $\lambda \notin \bar{H}_{i}$. For $x^{\prime} \in L_{i}$, there is $x \in K_{i}$ such that $x^{\prime}=x+M$. There are sequences $\left\{y_{n}\right\} \subset X_{T}\left(\bar{H}_{i}\right)$ and $\left\{z_{n}\right\} \subset M$ such that $y_{n}+z_{n} \rightarrow x$. Moreover, on $\mathbf{C}-\bar{H}_{i}$ there is an analytic function $f_{n}$ such that $y_{n}=(\lambda-T) f_{n}(\lambda), \lambda \notin \bar{H}_{i}$. Denote cosets in $K_{i} / M$ by $x^{\prime}$, etc. Then for each $\lambda \notin \bar{H}_{i},\left(\lambda-T^{M}\right) f_{n}(\lambda)^{\prime}=y_{n}^{\prime} \rightarrow x^{\prime}$. Fix $\lambda$ and suppose that $\left\{f_{n}(\lambda)^{\prime}\right\}$ does not converge. Then there is $p>0$ and a subsequence of $\left\{f_{n}(\lambda)^{\prime}\right\}$ which we write as $\left\{f_{k}(\lambda)^{\prime}\right\}$ such that $\left\|f_{k}(\lambda)^{\prime}-f_{k+1}(\lambda)^{\prime}\right\| \geq p$ for each $k$. But the corresponding subsequence 
$\left\{y_{k}^{\prime}\right\}$ converges to $x^{\prime}$, so $v_{k}=y_{k}^{\prime}-y_{k+1}^{\prime} \rightarrow 0$. Put $g_{l}(\lambda)=f_{k}(\lambda)^{\prime}-$ $f_{k+1}(\lambda)^{\prime}$; hence $\left(\lambda-T^{M}\right) g_{k}(\lambda)=v_{k} \rightarrow 0$. Since $M$ is strongly analytic, $g_{k}(\lambda) \rightarrow 0$, a contradiction. Thus $f_{n}(\lambda)^{\prime} \rightarrow w_{\lambda} \in L_{l}$. Clearly $x^{\prime}=$ $\left(\lambda-T^{M}\right) w_{\lambda}$, so $\lambda-T^{M}$ is surjective on $L_{l}$ for $\lambda \notin \bar{H}_{l}$. It follows that $\sigma\left(T^{M} \mid L_{l}\right) \subset \bar{H}_{l}$, so the theorem is proved.

4. Reflexive spaces. In order to get sharper results, we shall assume in this section that $X$ is reflexive except as otherwise indicated.

By Corollary 1 it is clear that the adjoint of a decomposable operator is quasi-decomposable. But since decomposable operators need not be strongly decomposable, it is not clear that such an operator is strongly quasi-decomposble. Our next theorem shows that this is nearly the case. First we have

Proposition 2. Let $X$ be reflexive. If $T^{*}$ is decomposable, then the restriction $T \mid X_{T}(G)^{-}$is quasi-decomposable for each $G$.

Proof. We first prove that if $G$ and $H$ are open, $X_{T}(G \cup H)^{-}=$ $X_{T}(G)^{-} \vee X_{T}(H)^{-}$. We compute

$$
\begin{aligned}
X_{T}(G \cup H)^{\perp} & =X_{T^{*}}^{*}[\mathbf{C}-(G \cup H)]=X_{T^{*}}^{*}(\mathbf{C}-G) \cap X_{T^{*}}^{*}(\mathbf{C}-H) \\
& =X_{T}(G)^{\perp} \cap X_{T}(H)^{\perp},
\end{aligned}
$$

where we use a consequence of Theorem 1 and the fact that $X_{T}(\cdot)$ preserves intersections. By reflexivity an equivalent version of (5) is

$$
X_{T}(G \cup H)^{-}=\left[X_{T}(G)^{-}+X_{T}(H)^{-}\right]^{-}=X_{T}(G)^{-} \vee X_{T}(H)^{-} .
$$

Now let $G$ be any open set and let $\left\{H_{1}, H_{2}\right\}$ be an open cover of $\mathbf{C}$. By (6)

$$
X_{T}(G)^{-}=X_{T}\left(H_{1} \cap G\right)^{-} \vee X_{T}\left(G \cap H_{2}\right)^{-} .
$$

Let $M=X_{T}(G)^{-}$and $S=T \mid M$. It is easy to prove that $M_{S}\left(\bar{H}_{l}\right)=M \cap$ $X_{T}\left(\bar{H}_{\imath}\right)$ is spectral maximal for $S$, and clearly $X_{T}\left(H_{\imath} \cap G\right)^{-} \subset M_{S}\left(\bar{H}_{\imath}\right) \subset$ $M$, hence $S$ is quasidecomposable, and the proof is complete.

Let us remark that condition (6) above is a necessary one for an (SQD) operator [15, Theorem 8.3].

THEOREM 6. For $T$ on reflexive $X$, the following are equivalent:

(i) $T$ is decomposable;

(ii) $T \mid X_{T}(G)^{-}$is quasi-decomposable for each open $G$ and (2) of Theorem 2 holds;

(iii) $T$ is quasi-decomposable and (2) holds. 
Proof. Clearly (ii) $\Rightarrow$ (iii), and (iii) $\Rightarrow$ (i) by Theorem 2. Finally (i) $\Rightarrow$ (ii) follows from Proposition 2.

Our results indicate that duality properties for (QD) operators may be hard to obtain without additional hypotheses like (2). We now seek alternative hypotheses which promote (ASD) to (D).

We noted above that a decomposable operator satisfies $(\beta)$. Earlier Bishop had proved that if $T$ and $T^{*}$ both have property $(\beta)$ on a reflexive space, $T$ has an (ASD) [6, Theorem 5]. More recently, the author sharpened this result as follows.

THEOREM 7 [19]. Let $X$ be reflexive. Then $T$ is decomposable iff $T$ and $T^{*}$ both satisfy $(\beta)$.

If $T$ alone satisfies ( $\beta$ ), it need not have an (ASD), e.g., the shift on Hilbert space. Nor is it known if $(\beta)$ and (ASD) together imply decomposability. However, if $T$ has (ASD) consisting of strongly analytic subspaces, then it is decomposable.

THEOREM 8. Let $X$ be reflexive. If $T$ has the (ASD) such that each invariant subspace may be chosen strongly analytic, then $T$ is decomposable.

First, observe that the hypotheses of Theorem 8 imply that $T$ has property $(\beta)$. For let $M$ be a strongly analytic subspace of $T$ in the (ASD) corresponding to $G$ in the cover $\{G, H\}$ of $\mathbf{C}$ such that $G \cap \sigma(T)$ $=\varnothing$. By [18, p. 19] $\sigma(T \mid M) \subset \sigma(T)$, so it follows that $\sigma(T \mid)=\varnothing$, hence $M=0$. Thus $T$ has property $(\beta)$.

The proof of Theorem 8 is broken into two lemmas, each having its own interest (we assume the hypotheses of Theorem 8).

Lemma 2. Let $F$ be closed in $\mathbf{C}$ and let $\left\{M_{\alpha}\right\}$ be a family of strongly analytic subspaces with $\sigma\left(T \mid M_{\alpha}\right) \subset F$ for each $\alpha$. If $M=\mathrm{V}_{\alpha} M_{\alpha}$, then $\sigma(T \mid M) \subset F$.

Proof. Since $T$ satisfies $(\beta) T$ and $T \mid M$ have the svep. Hence it suffices to prove that $\lambda-T$ is surjective on $M$ for $\lambda \notin F$ [8, Prop. 1.2]. Let $x \in M$. For each $n=1,2, \ldots$, there is $x_{n} \in M_{n}=M_{\alpha_{n}} \in\left\{M_{\alpha}\right\}$ such that $x_{n} \rightarrow x$. The corresponding sequence of analytic functions $f_{n}$ defined by $f_{n}(\mu)=R\left(\mu ; T \mid M_{n}\right) x_{n}$ on $\mathbf{C}-F$ has the property that $(\mu-T) f_{n}(\mu)$ $\rightarrow x$ uniformly on $\mathbf{C}-F$. We claim that $\left\{f_{n}\right\}$ is uniformly bounded on 
compact sets in $\mathbf{C}-F$. If not, there exists a compact $D \subset \mathbf{C}-F$ and (if necessary) a subsequence of $\left\{f_{n}\right\}$ also denoted $\left\{f_{n}\right\}$ such that $\left\|f_{n}\left(\mu_{n}\right)\right\| \geq n$ for some $\mu_{n} \in D$. Then clearly $(\mu-T)\left(n^{-1} f_{n}(\mu)\right) \rightarrow 0$ on $\mathbf{C}-F$. Since $T$ has property $(\beta), n^{-1} f_{n} \rightarrow 0$ uniformly on $D$, a contradiction. So for $\lambda \notin F$, since $X$ is reflexive $\left\{f_{n}(\lambda)\right\}$ has a subsequence converging weakly to $u \in X$. The convexity of $M$ implies $u \in M$, and weak continuity of $T$ implies $x=(\lambda-T) u$. Hence $\lambda-T$ is surjective on $M$ and $\sigma(T \mid M) \subset F$.

LEMMA 3. With the hypotheses of Lemma 2 and $F=\bar{G}$ for $G$ open, we also have $\sigma\left(T^{M}\right) \subset C-G$.

Proof. Let $G$ be open and let $\left\{H_{\alpha}\right\}$ be the family of all open $H_{\alpha}$ such that $\left\{G, H_{\alpha}\right\}$ covers $\mathrm{C}$ for each $\alpha$. (Note: $\cap_{\alpha} \bar{H}_{\alpha}=C-G$.) Without loss of generality, we may suppose that for each $\alpha$ there exist strongly analytic spaces $M_{\alpha}, N_{\alpha}$ such that $X=M_{\alpha} \vee N_{\alpha}$ and $\sigma\left(T \mid M_{\alpha}\right) \subset \bar{G}$ and $\sigma\left(T \mid N_{\alpha}\right) \subset$ $\bar{H}_{\alpha}$. We prove that $\sigma\left(T^{\alpha}\right) \subset \bar{H}_{\alpha}$ for each $\alpha$ where $T_{\alpha}$ is the induced operator on $X / M_{\alpha}$. Since $T^{\alpha}$ has the svep (each $\alpha$ ), we need only prove that $\lambda-T^{\alpha}$ is surjective for $\lambda \notin \bar{H}_{\alpha}$. For arbitrary $x \in X$, let $\left\{y_{n}\right\} \subset M_{\alpha}$ and $\left\{z_{n}\right\} \subset N_{\alpha}$ such that $y_{n}+z_{n} \rightarrow x$. On $\mathbf{C}-\bar{H}_{\alpha}$ the analytic functions $f_{n}$ defined by $f_{n}(\lambda)=R\left(\lambda ; T \mid N_{\alpha}\right) z_{n}$ satisfy

$$
\left\|x-y_{n}-(\lambda-T) f_{n}(\lambda)\right\| \rightarrow 0 \text { on } \mathbf{C}-\bar{H}_{\alpha} .
$$

In $X / M_{\alpha}$ with $x^{\prime}=x+M_{\alpha}$, etc.,

$$
\left\|x^{\prime}-\left(\lambda-T^{\alpha}\right) f_{n}^{\prime}(\lambda)\right\| \rightarrow 0 \quad \text { on } \mathbf{C}-\bar{H}_{\alpha} .
$$

As in the proof of Lemma $2, \mathrm{x}^{\prime}=\left(\lambda-T^{\alpha}\right) w$ for some $w \in X / M_{\alpha}$. So $\sigma\left(T^{\alpha}\right) \subset \bar{H}_{\alpha}$ for each $\alpha$.

Moreover, since $\sigma\left(T^{\alpha}\right)=\sigma\left(T^{*} \mid M_{\alpha}{ }^{\perp}\right)$ for each $\alpha$, if $M=\mathrm{V}_{\alpha} M_{\alpha}$ it follows that $\sigma\left(T^{*} \mid M^{\perp}\right) \subset \sigma\left(T^{*} \mid M_{\alpha}^{\perp}\right) \subset \bar{H}_{\alpha}$, all $\alpha$. Hence $\sigma\left(T^{M}\right)=$ $\sigma\left(T^{*} \mid M^{\perp}\right) \subset \cap \bar{H}_{\alpha}=\mathrm{C}-G$, and Lemma 2 is proved.

To prove Theorem 8, merely apply Lemmas 2 and 3 and Theorem 1 (iii).

COROllary 3. On a reflexive space, the following are equivalent:

(i) $T^{*}$ is strongly decomposable;

(ii) $T$ is quasi-decomposable and $X_{T}(G)^{-}$is strongly analytic for each open $G$.

(iii) $T^{*}$ is strongly quasi-decomposable and each of its spectral maximal spaces is strongly analytic. 
Proof. (i) $\Leftrightarrow$ (ii). Clearly (i) $\Rightarrow$ (ii) by Theorem 5. For the converse, use Theorems 8 and 5. (i) $\Leftrightarrow$ (iii). If (i) holds, then $T^{* M}$ is decomposable for each spectral maximal $M$ for $T^{*}$ [4], so each such $M$ is strongly analytic for $T^{*}$. For the converse, apply Theorem 8 .

REMARK. Assertion (ii) of Corollary 3 is similar to a recent result of Snader [26, Theorem 3.1].

5. Strongly bi-decomposable operators. It has already been observed that $(\mathrm{D}) \Rightarrow(\mathrm{SD})$ may fail and strong decomposability may not be preserved under duality. But these counter-examples are defined on nonreflexive Banach spaces. Hence the questions (D) $\Rightarrow(\mathrm{SD})$ and (SD) $\Rightarrow$ $(\mathrm{SD})^{*}$ are still open for operators on reflexive spaces. In this section, we present some results which might be pertinent to these questions. To simplify terminology, we call $T$ strongly bi-decomposable if both $T$ and $T^{*}$ are strongly decomposable. By [22, Cor. 3] $T^{*}$ is strongly bi-decomposable if $T^{* * *}$ is.

We begin with an observation.

Proposition 3. If $(\mathrm{QD}) \Rightarrow(\mathrm{D})$ on reflexive spaces, then $T^{M}$ is decomposable if $T$ is decomposable and $M$ is spectral maximal. In addition, if $(\mathrm{D}) \Rightarrow(\mathrm{SQD})$, then every decomposable operator is strongly bi-decomposable.

Proof. Let $T$ be decomposable with spectral maximal space $M$. By Theorem $6, T^{*}$ is quasi-decomposable on $M^{\perp}=X_{T^{*}}^{*}(G)^{-}$for some open $G$. By hypothesis this restriction $T^{*} \mid M^{\perp}$ is decomposable, hence $T^{M}$ is decomposable by Corollary 1 . If the hypothesis (D) $\Rightarrow$ (SQD) holds, then each restriction of $T$ to a spectral maximal $M$ is quasi-decomposable, so $T \mid M$ is also decomposable. Hence $T$ is strongly decomposable, and similarly $T^{*}$ is strongly decomposable.

Thus the conclusion of Theorem 6 (ii) is not quite strong enough to ensure strong bi-decomposability. Our next theorem gives criteria for this property. If $G$ is open and $F$ is closed, let $T^{G}\left[T^{F}\right]$ denote the operator induced by $X_{T}(G)^{-}\left[X_{T}(F)\right]$.

THEOREM 9. Let $X$ be reflexive. Then the following are equivalent:

(i) $T$ is strongly bi-decomposable.

(ii) For each open $G$ and closed $F, T^{G}$ is decomposable and $T^{F}$ is strongly decomposable. 
(iii) $T$ is (SQD) and for each open $G$ and closed $F$ subspaces $X_{T}(G)^{-}$ and $X_{T}(F)$ are strongly analytic.

(iv) $T$ is decomposable and for each open $G$ the subspace $X_{T}(G)^{-}$ $\left[X_{T^{*}}^{*}(G)^{-}\right]$is strongly analytic for $T\left[T^{*}\right]$.

Proof. The equivalence (i) $\Leftrightarrow$ (iv) follows from Theorem 5. If (i) holds, then $T^{*}\left|X_{T^{*}}^{*}(\mathbf{C}-G)=T^{*}\right| X_{T}(G)^{\perp}$ is decomposable. Hence $T^{G}$ is decomposable by Corollary 1 . Since $T$ is strongly decomposable also, $T^{F}$ is strongly decomposable by [4, Th. 1.8]. (ii) $\Leftrightarrow$ (iii). If $T^{G}$ and $T^{F}$ are decomposable, then $X_{T}(G)^{-}$and $X_{T}(F)$ are strongly analytic by a result of Foias [12]. Since $T^{F}$ is also strongly decomposable, (iii) follows by taking $F=\varnothing$. If (iii) is true, then $T \mid X_{T}(F)$ is quasidecomposable, so $T$ is strongly decomposable by Theorem 8 . Since each $X_{T}(G)^{-}, G$ open, is strongly analytic, $T^{*}$ is strongly decomposable by Theorem 5 . Hence (iii) $\Leftrightarrow$ (i) and the proof is complete.

We next consider some necessary conditions for an operator to be strongly bi-decomposable. We say $T$ satisfies $(\alpha)$ if the following holds:

( $\alpha)$ if $\sigma(T)$ has more than one point, there exist nonzero strongly analytic subspaces $M_{1}$ and $M_{2}$ such that $\sigma\left(T \mid M_{i}\right)$ are disjoint.

Proposition 4. Let $X$ be reflexive. If $T$ is strongly decomposable, then $T$ and $T^{*}$ both satisfy $(\alpha)$.

Proof. If $\sigma(T)$ has only one point, then $T$ and $T^{*}$ have property $(\alpha)$ vacuously. In case $\sigma(T)$ has more than one point, there exist open sets $G_{1}$, $G_{2}$ with disjoint closures such that $\sigma(T) \cap G_{i} \neq \varnothing(i=1,2)$. Hence $M_{l}=X_{T}\left(\bar{G}_{\imath}\right)$ are spectral maximal spaces such that $\sigma\left(T \mid M_{1}\right) \cap \sigma\left(T \mid M_{2}\right)$ $=\varnothing$. Since $M_{i}$ are both strongly analytic (Cor. 3), $T$ satisfies $(\alpha)$. Also by Corollary 3 , each $X_{T^{*}}^{*}(G)^{-}$is strongly analytic for each open $G$, so $T^{*}$ has property $(\alpha)$ by similar reasoning.

Proposition 5. If $X$ is reflexive, then T satisfies $(\beta)$ if it satisfies $(\alpha)$.

Proof. Let $M$ and $N$ be nonzero subspaces in $(\alpha)$. We claim $M+N$ is direct. If so, $M+N$ is closed, hence by [18, Prop. 7] the intersection $M \cap N=(0)$ is strongly analytic, i.e., $T$ satisfies $(\beta)$. To prove our claim, put $L=M \vee N$ and $S=T \mid L$. Then $\sigma\left(S^{N}\right) \subset \sigma(T \mid M)$. This last assertion is proved just as in the proof of Lemma 3. Since $M$ and $N$ are 
strongly analytic for $S$ as well as $T$, we obtain the inclusions

$$
\begin{aligned}
\sigma(S \mid M) \cup \sigma(S \mid N) & \subset \sigma(S) \subset \sigma\left(S^{N}\right) \cup \sigma(S \mid N) \\
& \subset \sigma(S \mid M) \cup \sigma(S \mid N),
\end{aligned}
$$

which prove that $\sigma(S)$ is the disjoint union of $\sigma(S \mid M)$ and $\sigma(S \mid N)$. Let $P$ be the Riesz projection on $L$ corresponding to the (Dunford) spectral set $\sigma(S \mid M)$. We hve

$$
\sigma(S \mid P L)=\sigma(S \mid M) ; \sigma(S \mid(I-P) L)=\sigma(S \mid N)
$$

hence $M \subset P L$ and $N \subset(I-P) L$ since these ranges are spectral maximal for $S$ [7, p. 17]. Clearly $M+N$ is a direct sum, and the proof is complete.

We collect these results in

THEOREM 10. Let $X$ be reflexive and consider the following statements:

(i) $T$ is strongly bi-decomposable;

(ii) either $T$ or $T^{*}$ is strongly decomposable;

(iii) $T$ and $T^{*}$ both satisfy $(\alpha)$;

(iv) $T$ and $T^{*}$ both satisfy $(\beta)$;

(v) $T$ and $T^{*}$ are both decomposable.

Then (i) $\Rightarrow$ (ii) $\Rightarrow$ (iii) $\Rightarrow$ (iv) $\Leftrightarrow$ (v).

Hence if $(D) \Rightarrow(S D)$ on reflexive spaces, (i)-(v) are equivalent. The existence of a counter-example would then be assured by a failure of any converse of the first three implications.

For completeness, we now discuss a condition sufficient for an operator to be strongly bi-decomposable (see (8) below). We first show that this condition is also sufficient for the following criterion due to Wang [27].

Proposition 6. Let $X$ be arbitrary. Then $T$ [resp. $\left.T^{*}\right]$ is strongly decomposable iff for each two open sets $G$ and $H$

$$
\begin{gathered}
X_{T^{*}}^{*}(G \cup H)^{W} \subset X_{T^{*}}^{*}\left(G_{\varepsilon}\right)^{W}+X_{T^{*}}^{*}(H)^{W} \\
{\left[\operatorname{resp.} X_{T}(G \cup H)^{-} \subset X_{T}\left(G_{\varepsilon}\right)^{-}+X_{T}(H)^{-}\right]}
\end{gathered}
$$

where $A^{W}$ denotes weak ${ }^{*}$ closure of $A$ and $G_{\varepsilon}$ is the $\varepsilon$-neighborhood of $\bar{G}$.

Proposition 7. Let $T$ be decomposable on the Banach space $X$. Suppose that for each closed $F$ and open $G$

$$
X_{T}(F)+X_{T}(G)^{-} \text {is norm closed in } X .
$$

If $T=S^{*}$ for some $S$, then (7a) holds; (7b) holds in any case. 
Proof. We prove (7a); the proof of (7b) is similar. Let $\varepsilon>0$ and let $K$ be open such that $G \subset K \subset \bar{K} \subset G_{\varepsilon}$. For an arbitrary $z \in X_{T}(G \cup H)^{W}$, let $\left\{x_{\alpha}\right\} \subset X_{T}(G \cup H)$ be a net converging weak* to $z$. Since $T$ has "almost localized spectrum" [24], for each $\alpha$ there are $u_{\alpha} \in X_{T}(H)$ and $v_{\alpha} \in X_{T}(G)$ with $x_{\alpha}=u_{\alpha}+v_{\alpha}$. Clearly $x_{\alpha} \in X_{T}(H)^{-}+X_{T}(\bar{K})$. By (8) the last manifold is closed, so by the closed graph theorem, there is $R>0$ such that for each $\alpha$ we have $u_{\alpha}^{\prime}, v_{\alpha}^{\prime}$ in $X_{T}(H)^{-}, X_{T}(\bar{K})$ resp. such that $x_{\alpha}=u_{\alpha}^{\prime}+v_{\alpha}^{\prime}$ and

$$
\left\|u_{\alpha}^{\prime}\right\|+\left\|v_{\alpha}^{\prime}\right\| \leq R\left\|x_{\alpha}\right\| .
$$

Since $\left\|x_{\alpha}\right\|$ is uniformly bounded, we can find subnets of $\left\{u_{\alpha}^{\prime}\right\}$ and $\left\{v_{\alpha}^{\prime}\right\}$ converging weak* to $u_{0}, v_{0} \in X$. Clearly $z=u_{0}+v_{0} \in X_{T}(H)^{W}+$ $X_{T}\left(G_{\varepsilon}\right)^{W}$, and (7a) is proved.

THEOREM 11. Let $X$ be reflexive. If $T$ is decomposable and (8) holds, then $T$ is strongly bi-decomposable.

Proof. By Propositions 6 and 7, $T^{*}$ is strongly decomposable. If $F$ is closed and $G$ is open, since $X$ is reflexive, we have the relations

$$
X_{T^{*}}^{*}(F)=X_{T}(\mathbf{C}-F)^{\perp} \quad \text { and } \quad X_{T^{*}}^{*}(G)^{-}=X_{T}(\mathbf{C}-G)^{\perp} .
$$

By (8) $X_{T}(\mathbf{C}-G)+X_{T}(\mathbf{C}-F)^{-}$is closed, hence $X_{T^{*}}^{*}(F)+X_{T^{*}}^{*}(G)^{-}$is also closed by [16, Lemma 4.9, p. 221]. Again, $T$ is strongly decomposable by Propositions 6 and 7.

In fact, Theorem 11 can be generalized as follows. Let $T$ satisfy (8) on arbitrary $X$. As before, $X_{T^{*}}^{*}(F)=X_{T}(\mathbf{C}-F)^{\perp}$, but now $X_{T}(\mathbf{C}-G)^{\perp}=$ $X_{T^{*}}^{*}(G)^{W}$. Then $X_{T^{*}}^{*}(F)+X_{T^{*}}^{*}(G)^{W}$ is norm closed [16, p. 221] and the proof of (7a) proceeds as in Proposition 7.

We mention finally that R. Evans [9] has studied "boundedly decomposable" operators. Each such operator satisfies (8) and is therefore strongly bi-decomposable by the last remark. Details are left to the reader.

\section{REFERENCES}

[1] E. Albrecht, A weakly decomposable operator which is not decomposable, Rev. Roum. Math. Pures Appl., 20 (1975), 855-861.

[2] On two questions of I. Colojoara and C. Foias, Manuscripta Math., 25 (1978), $1-15$.

[3] $\frac{1}{1-10}$, On decomposable operators, Integral Equations Operator Theory, 2 (1979), 
[4] C. Apostol, Restrictions and quotients of decomposable operators in a Banach space, Rev. Roum. Math. Pures Appl., 13 (1968), 147-150.

[5] _ Roots of decomposable operator valued analytic functions, Rev. Roum. Math. Pures Appl., 13 (1968), 433-438.

[6] E. Bishop, $A$ duality theorem for an arbitrary operator, Pacific J. Math., 9 (1959), 379-397.

[7] I. Colojoară and C. Foias, Theory of Generalized Spectral Operators, Gordon and Breach, New York, 1968.

[8] I. Erdelyi and R. Lange, Spectral Decompositions on Banach Spaces, Lecture Notes in Math., Springer, New York, 1977.

[9] R. Evans, Boundedly decomposable operators and the continuous functional calculus, Rev. Roum. Math. Pures Appl., 28 (1983), 465-473.

[10] J. Finch, The single valued extension property on a Banach space, Pacific J. Math., 58 (1975), 61-69.

[11] C. Foias, Spectral maximal spaces and decomposable operators, Arch. Math. (Basel), 14 (1963), 341-349.

[12] , On the maximal spectral spaces of a decomposable operator, Rev. Roum. Math. Pures Appl., 15 (1970), 1599-1606.

[13] S. Frunză, A duality theorem for decomposable operators, Rev. Roum. Math. Pures Appl., 16 (1971), 1055-1058.

[14] _ The single valued extension property for coinduced operators, Rev. Roum. Math. Pures Appl., 18 (1973), 1061-1065.

[15] A. Jafarian, Weak and quasi-decomposable operators, Rev. Roum., Math. Pures Appl., 22 (1977), 195-212.

[16] T. Kato, Perturbation Theory for Linear Operators, Springer, New York, 1966.

[17] R. Lange, Analytically decomposable operators, Trans. Amer. Math. Soc., 244 (1978), $225-240$.

[18] Strongly analytic subspaces, in Operator theory and functional analysis, I. Erdelyi, ed., Pitman, San Francisco, 1979.

[19] _ A purely analytic criterion for a decomposable operator, Glasgow Math. J., 21 (1980), 69-70.

[20] - On generalization of decomposability, Glasgow Math. J., 22 (1981), 77-81.

[21] _ Equivalent conditions for a decomposable operator, Proc. Amer. Math. Soc., 82 (1981), 401-406.

[22] G. Liu and S. Wang, On the duality theorem for S-decomposable operators, J. Math. Analysis Appl., to appear.

[23] B. Nagy, Operators with the spectral decomposition property are decomposable, Studia Sci. Math. Hungar., 13 (1978), 429-432.

[24] M. Radjabalipour, Equivalence of decomposable and 2-decomposable operators, Pacific J. Math., 77 (1978), 243-247.

[25] G. Shulberg, Decomposable restrictions and extensions, J. Math. Analysis Appl., 83 (1981), 144-158.

[26] J. Snader. Strongly analytic subspaces and strongly decomposable operators, Pacific J. Math., to appear.

[27] S. Wang, A characterization of strongly decomposable operators, Amer. Math. Soc. Abstracts, 4 (1983), No. 83T-47 - 416.

Received December 9, 1983. 


\section{PACIFIC JOURNAL OF MATHEMATICS EDITORS}

V. S. VARADARAJAN (Managing Editor)
University of California
Los Angeles, CA 90024
HEBERT CLEMENS
University of Utah
Salt Lake City, UT 84112
CHARLES R. DEPRIMA
California Institute of Technology
Pasadena, CA 91125

\section{R. FINN}

Stanford University

Stanford, CA 94305

HeRmanN FlaschKa

University of Arizona

Tucson, AZ 85721

RAMESH A. GANGOLli

University of Washington

Seattle, WA 98195

ROBION KIRBY

University of California

Berkeley, CA 94720

\section{C. MoOre}

University of California

Berkeley, CA 94720

H. SAMELSON

Stanford University

Stanford, CA 94305

HAROLD STARK

University of California, San Diego

La Jolla, CA 92093

ASSOCIATE EDITORS
R. ARENS
E. F. BECKENBACH
B. H. NeUmanN
F. WOLF
K. Yoshida (1906-1982)

\section{SUPPORTING INSTITUTIONS}

UNIVERSITY OF ARIZONA

UNIVERSITY OF BRITISH COLUMBIA

CALIFORNIA INSTITUTE OF TECHNOLOGY

UNIVERSITY OF CALIFORNIA

MONTANA STATE UNIVERSITY

UNIVERSITY OF NEVADA, RENO

NEW MEXICO STATE UNIVERSITY

OREGON STATE UNIVERSITY
UNIVERSITY OF OREGON

UNIVERSITY OF SOUTHERN CALIFORNIA

STANFORD UNIVERSITY

UNIVERSITY OF HAWAII

UNIVERSITY OF TOKYO

UNIVERSITY OF UTAH

WASHINGTON STATE UNIVERSITY

UNIVERSITY OF WASHINGTON 


\section{Pacific Journal of Mathematics}

\section{Vol. 121, No. $1 \quad$ November, 1986}

Om P. Agrawal, Douglas Napier Clark and Ronald George Douglas,

Invariant subspaces in the polydisk $\ldots \ldots \ldots \ldots \ldots \ldots \ldots \ldots \ldots \ldots$

Christoph Bandt and Gebreselassie Baraki, Metrically invariant measures

on locally homogeneous spaces and hyperspaces $\ldots \ldots \ldots \ldots \ldots \ldots \ldots$

Marcy Mason Barge, Horseshoe maps and inverse limits ..............29

Russell Gene Bilyeu, Robert Richard Kallman and Paul Weldon Lewis,

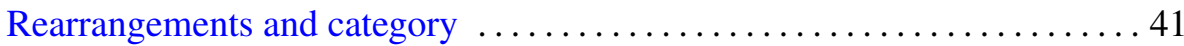

Jean Bourgain, A problem of Douglas and Rudin on factorization . . .......47

Hernan Cendra, A normal form and integration in finite terms for a class of

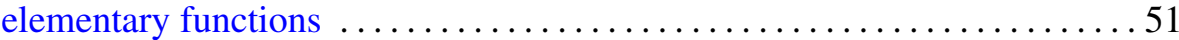

Ky Fan, The angular derivative of an operator-valued analytic function . . . . 67

Gerhard Gierz, On the Dunford-Pettis property of function modules of

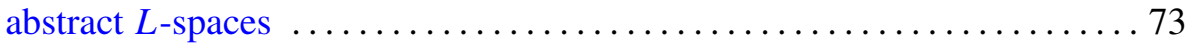

Gabriel Katz, On polynomial generators in the algebra of complex functions

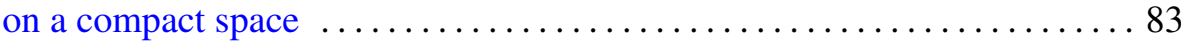

Ridgley Lange, Duality and asymptotic spectral decompositions $\ldots \ldots \ldots . .93$

Anthony To-Ming Lau and Peter F. Mah, Quasinormal structures for certain spaces of operators on a Hilbert space ................... 109

R. Daniel Mauldin, Correction: "The set of continuous nowhere differentiable functions"

Alan Harvey Mekler and Saharon Shelah, $\omega$-elongations and Crawley's problem

Alan Harvey Mekler and Saharon Shelah, The solution to Crawley's problem

Richard Rochberg, Deformation of uniform algebras on Riemann surfaces

Joseph Roitberg, On weak epimorphisms in homotopy theory

Jesús M. Ruiz, A remark on fields with the dense orbits property

Henry Wente, Counterexample to a conjecture of H. Hopf

David G. Wright, Rigid sets in $E^{n}$ 